(adjusted Odds Ratio 1.33, 95\% CI 1.15-1.55) compared to permanent day workers. ORs increased with cumulative years of rotating shift work and the OR for more than 30 years work 1.54 (1.22-1.94). Having ever worked in permanent night shift was not associated with colorectal cancer risk. Analysis on geneenvironment interactions with genes in circadian, melatonin and sleep pathways are ongoing and will be presented.

Conclusions In this large population based study we found an increase in colorectal cancer risk associated with rotating shift work.

\section{LONGITUDINAL MEASUREMENT OF WORK STRESSORS IN PREGNANCY}

1,2John D Meyer, ${ }^{2}$ Nicholas Warren, ${ }^{2}$ Ginger Nichols. 'ICahn-Mount Sinai School of Medicine, New York, NY, USA; ${ }^{2}$ University of Connecticut Health Center, Farmington, CT, USA

\subsection{6/oemed-2014-102362.17}

Objectives Pregnancy represents a dynamic period when both work conditions and worker assessment of occupational psychosocial stressors may change. We undertook longitudinal repeated measurements of job control, efforts, reward, and overcommitment in pregnant working women to assess direction and magnitude of change across pregnancy.

Method The Job Content and Effort-Reward Imbalance (ERI) Questionnaires were given to 61 working pregnant women recruited at 8-12 weeks gestation and repeated 3 more times across the pregnancy. Demographic data, self-rated (SR) health and stress measures, and blood pressure (BP) data were also collected at each visit. Results were analysed using multilevel linear regression models and generalised estimating equations.

Results 56 subjects (91\%) completed at least two waves; 42 (69\%) completed all four. Progressive declines in job efforts, rewards, and overcommitment were noted across pregnancy; ERI remained stable. Job Control increased. Black subjects showed evidence of lower job control but also lower effortreward imbalance across pregnancy, contrasted with Whites/Hispanics. Overcommitment showed the strongest negative association with indices of maternal health, including BP, and SR stress and health. The strongest associations of job constructs with maternal health were noted at mid-second trimester (20-24 weeks) with a decline thereafter.

Conclusions Correlations between occupational psychosocial stressors and intermediate pregnancy-related outcome variables are greatest in early-to-mid-pregnancy. Declining effort and increased control across pregnancy suggest that, in this group, work conditions may be improved, either by the employer or worker, as pregnancy progresses. The possibility of a critical period when associations between work stressors and pregnancy outcomes is also suggested by these results.

\section{PARTICLE SIZE DISTRIBUTION IN ALUMINIUM MANUFACTURING FACILITIES}

${ }^{1}$ Sa Liu, ${ }^{1}$ Elizabeth M Noth, ${ }^{2}$ Christine Dixon-Ernst, ${ }^{1}$ Ellen A Eisen, ${ }^{3}$ Mark R Cullen, 'S Katharine Hammond. 'Division of Environmental Health Sciences, School of Public Health, University of California, Berkeley, CA, USA; ${ }^{2}$ Alcoa, Pittsburgh, PA, USA; ${ }^{3}$ Department of Internal Medicine, Stanford University, Stanford, CA, USA

\subsection{6/oemed-2014-102362.18}

Objectives As part of exposure assessment for an ongoing epidemiologic study of heart disease and fine particle exposures in aluminium manufacturing, area particle samples were collected in smelters and fabrication facilities to assess instrument reliability and particle size distribution at different process areas. Method Personal Modular Impactors (PMI) and Minimicro-orifice uniform deposition impactors (MiniMOUDI) were used to collect samples. The coefficient of variation (CV) of collocated samples of the same type was used to evaluate the reproducibility of the impactors. $\mathrm{PM}_{2.5}$ measured by PMI was compared to $\mathrm{PM}_{2.5}$ calculated from MiniMOUDI data to assess the validity of using PMI to measure fine particles in personal sampling. Mass median aerodynamic diameter (MMAD) was calculated to characterise particle size distribution at different locations.

Results 62 MiniMOUDI and 71 PMI samples were collected at 44 production areas. Most of CVs were less than 30\%. The slope of the liner regression of PMI_PM PM.5 $_{2}$ versus MiniMOUDI PM $\mathrm{PM}_{2.5}$ was $1.12 \mathrm{mg} / \mathrm{m}^{3}$ per $\mathrm{mg} / \mathrm{m}^{3}$ ( \pm 0.05$)$, with correlation coefficient of 0.97 ( \pm 0.01$)$. MMADs in fabrications were significantly smaller and less variable than those in smelters $(\mathrm{p}=$ 0.001). The fraction of $\mathrm{PM}_{10}$ which was $\mathrm{PM}_{1.0}$ or $\mathrm{PM}_{0.56}$ was significantly higher in fabrications than in smelters $(\mathrm{p}<0.001)$.

Conclusions The reproducibility for impactors was moderate to high. $\mathrm{PM}_{2.5}$ measured by PMI can be a valid measure for fine particle exposure in personal sampling. The concentrations of submicron and quasi-ultrafine particles were similar in fabrications and smelters. $\mathrm{PM}_{2.5}$ is not a good surrogate for ultrafine particles in this setting.

\section{INCIDENCE OF KNEE PAIN AND ITS WORK-RELATED RISK FACTORS IN A LARGE WORKING POPULATION}

${ }^{1,2}$ Eléonore Herquelot, ${ }^{1,2}$ Annette Leclerc, ${ }^{3}$ Yves Roquelaure, ${ }^{3}$ Julie Bodin, ${ }^{4}$ Catherine $\mathrm{Ha}$, 1,2 Diane Cyr, ${ }^{1,2}$ Marcel Goldberg, ${ }^{1,2}$ Marie Zins, ${ }^{2,5}$ Alexis Descatha. ${ }^{1}$ Inserm, Centre for Research in Epidemiology and Population Health (CESP), U1018, Population-Based Epidemiological Cohorts Research Platform, Villejuif, France; ${ }^{2}$ University Versailles StQuentin, Versailles, France; ' ${ }^{3}$ LUNAM Université, Université d'Angers, Laboratoire D'ergonomie Et D'épidémiologie en Santé Au Travail (LEEST), Angers, France; ${ }^{4}$ Institut de Veille Sanitaire (InVS), Département Santé Travail, Saint-Maurice, France; ${ }^{5} A P-H P$, Poincaré University Hospital, Occupational Health Unit, Garches, France

\subsection{6/oemed-2014-102362.19}

Objectives This study aims to estimate the incidence of knee pain and its risk factors in a general French working population, representative of the work force.

Method Of 3710 workers of a French region included in a study in 2002-2005 with a self-administered questionnaire, 2332 completed a follow-up questionnaire in 2007-2011. The questionnaires included musculoskeletal symptoms, individual and occupational exposures, and physical limitations (at followup only). Incident knee pain in 2007-2011 (i.e. subjects not suffering of knee pain at baseline and suffering of knee pain at follow-up) was dichotomized into sub-chronic knee pain (1-29 days) and chronic knee pain ( $>30$ days). Associations between the incident knee pains and individual and work-related risk factors at baseline were studied separately by gender, using multinomial logistic regressions.

Results Of the 1616 respondents without knee pain at baseline, $122(7.5 \%)$ reported chronic knee pain and $243(15.0 \%)$ reported sub-chronic knee pain; 43\% of workers with incident chronic knee pain and $30 \%$ of workers with incident sub-chronic knee pain had other chronic pains at baseline, and respectively $51 \%$ and $28 \%$ reported limitations in climbing stairs. After adjustment for age and BMI, significant associations were found between incident knee pain and handling loads $>4 \mathrm{~kg}$ (OddsRatio (OR) 2.1 (1.2-3.6) among men, OR 2.3 (1.1-5.0) among 